\title{
Population transcriptomics of Drosophila melanogaster females
}

Lena Müller ${ }^{1}$, Stephan Hutter ${ }^{1}$, Rayna Stamboliyska ${ }^{1}$, Sarah S Saminadin-Peter ${ }^{1,2}$, Wolfgang Stephan ${ }^{1}$, John Parsch ${ }^{1 *}$

\begin{abstract}
Background: Variation at the level of gene expression is abundant in natural populations and is thought to contribute to the adaptive divergence of populations and species. Gene expression also differs considerably between males and females. Here we report a microarray analysis of gene expression variation among females of 16 Drosophila melanogaster strains derived from natural populations, including eight strains from the putative ancestral range in sub-Saharan Africa and eight strains from Europe. Gene expression variation among males of the same strains was reported previously.

Results: We detected relatively low levels of expression polymorphism within populations, but much higher expression divergence between populations. A total of 569 genes showed a significant expression difference between the African and European populations at a false discovery rate of $5 \%$. Genes with significant overexpression in Europe included the insecticide resistance gene Cyp6g1, as well as genes involved in proteolysis and olfaction. Genes with functions in carbohydrate metabolism and vision were significantly over-expressed in the African population. There was little overlap between genes expressed differently between populations in females and males.

Conclusions: Our results suggest that adaptive changes in gene expression have accompanied the out-of-Africa migration of $D$. melanogaster. Comparison of female and male expression data indicates that the vast majority of genes differing in expression between populations do so in only one sex and suggests that most regulatory adaptation has been sex-specific.
\end{abstract}

\section{Background}

Over the past decade, microarray studies have shown that variation at the level of gene expression is abundant within natural populations $[1,2]$. Similar studies have also revealed extensive differences in gene expression between males and females [3]. Indeed, in the wellstudied model organism Drosophila melanogaster, genes that differ in expression between the sexes (sex-biased genes) greatly outnumber those that differ in expression between individuals of the same sex [4-6]. Thus, it is important to account for sex when characterizing gene expression variation within species.

To date, most studies of gene expression variation within Drosophila species have been limited to a small number of laboratory strains, or to strains derived from

\footnotetext{
* Correspondence: parsch@zi.biologie.uni-muenchen.de

'Department of Biology II, University of Munich (LMU), 82152 PlaneggMartinsried, Germany

Full list of author information is available at the end of the article
}

a single non-African population [4-8]. These studies are useful for determining the amount and underlying genetic architecture of gene expression variation among individuals, but reveal little about the potential for gene expression levels to evolve adaptively in response to local environmental conditions. Studies of genomic and mitochondrial DNA variation suggest that $D$. melanogaster expanded from its ancestral range in sub-Saharan Africa and began to colonize Europe about 15,000 years ago [9-13], with a subsequent colonization of North America occurring within the past 500 years [14]. Presumably, the out-of-Africa expansion was accompanied by adaptation to the new, temperate environment, and several studies have provided evidence for genetic adaptation in derived D. melanogaster populations [11,15-17].

A previous microarray analysis of male gene expression variation in eight $D$. melanogaster strains from the ancestral species range (Zimbabwe, Africa) and eight
C Biomed Central

C 2011 Müller et al; licensee BioMed Central Ltd. This is an Open Access article distributed under the terms of the Creative Commons Attribution License (http://creativecommons.org/licenses/by/2.0), which permits unrestricted use, distribution, and reproduction in any medium, provided the original work is properly cited. 
strains from Europe (the Netherlands) identified 153 genes with a significant expression difference between the populations [18]. These genes represent candidates for those having undergone adaptive regulatory evolution in response to the local environment and were enriched for genes with functions in insecticide resistance, fatty acid metabolism, and flight [18]. The male expression data, however, provide only half of the story. Given the extent of sex-biased gene expression in D. melanogaster $[19,20]$, the potential for differences in the mode of inheritance of gene expression between males and females [21], the impact of the Y chromosome on gene expression variation [22,23], and the proposed differences in effective population size between males and females of the African and European populations $[24,25]$, it is desirable to investigate expression variation among females of the same populations.

Here we report a microarray survey of gene expression variation in adult females of the African and European D. melanogaster populations. Our analyses are performed on three levels. First, we use the new microarray data to determine levels of gene expression polymorphism among females of each population, as well as gene expression divergence between populations. Second, we examine the contribution of sex-biased genes to the observed patterns of expression polymorphism and divergence. Third, we compare the female results with previously published results from males in order to detect differences in expression variation between the sexes. We find that, in females, there is little gene expression polymorphism within populations, but a relatively large number of genes with a significant expression difference between populations. The latter represent candidates for population-specific gene regulatory evolution and several of these genes show evidence that positive selection has acted on linked, cis-regulatory sequences. We find that sex-biased genes do not make a disproportionate contribution to expression variation among females. A comparison of the female and male results suggests that substantial sex-specific adaptation of gene expression levels has occurred following the out-of-Africa migration of $D$. melanogaster.

\section{Results and Discussion}

\section{Gene expression polymorphism}

We analyzed gene expression variation among adult females of 16 strains of $D$. melanogaster (eight from Zimbabwe, Africa and eight from the Netherlands, Europe) using CDMC 14kv1 whole-genome microarrays (Figure 1). The microarray features 14,439 unique $D$. melanogaster probes corresponding to 13,688 unique protein-coding genes. After quality control, we detected expression of 6,578 probes corresponding to 6,308 unique genes in all $16 \mathrm{D}$. melanogaster strains. Of these,

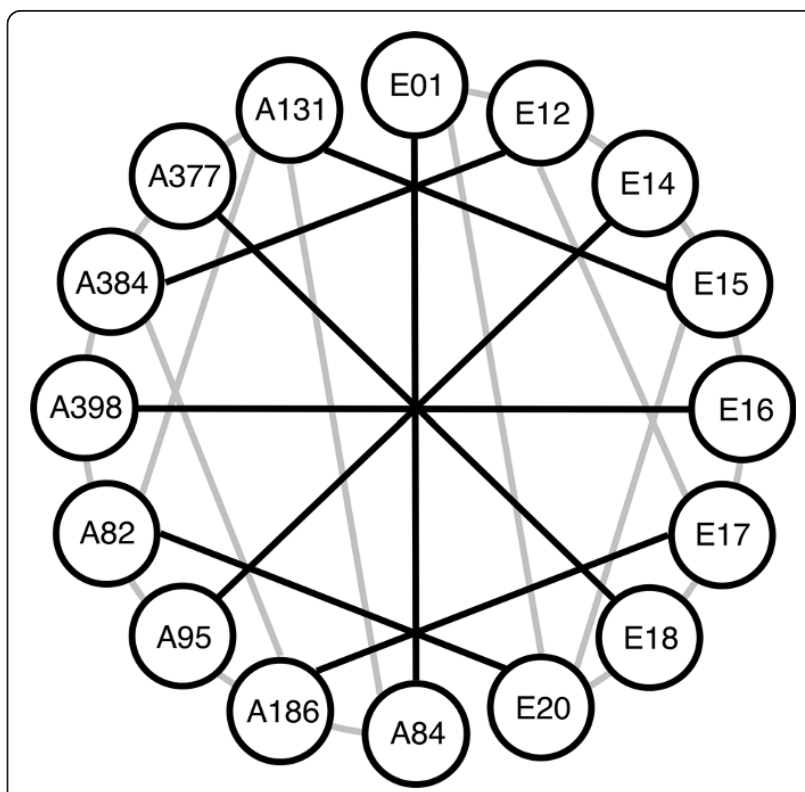

Figure 1 Microarray hybridization scheme. Each node represents one $D$. melanogaster strain, with ' $E$ ' indicating European and ' $A$ ' indicating African strains. Each line represents two microarray hybridizations (dye-swap replicates), with black indicating betweenpopulation and gray indicating within-population hybridizations.

$1,536(24 \%)$ showed a significant expression difference between at least two of the 16 strains at a nominal $P$-value of 0.001 , which corresponds to a FDR of $30 \%$. Overall, there was greater expression polymorphism among African strains than among European strains, but the greatest number of expression differences was found in comparisons between African and European strains (Table 1).

Across all 16 D. melanogaster strains, we found significantly less expression polymorphism in females than what was previously reported for males of the same strains [18], with females having 1.7 -fold fewer polymorphic genes ( $24 \%$ vs. $\left.38 \% ; \chi^{2}=230, P<0.0001\right)$, and 3.7-fold fewer significant pairwise differences per gene as males (0.89 vs. 3.28; Mann-Whitney test, $P<0.0001)$.

Table 1 Expression polymorphism within and between populations

\begin{tabular}{lccc}
\hline Comparison & $\begin{array}{c}\text { Number of } \\
\text { polymorphic } \\
\text { genes }\end{array}$ & $\begin{array}{c}\text { Mean } \\
\text { differences } \\
\text { per pairwise } \\
\text { comparison }\end{array}$ & $\begin{array}{c}\text { Mean } \\
\text { pairwise } \\
\text { differences } \\
\text { per } \\
\text { gene (in \%) }\end{array}$ \\
\hline Among all strains & 1536 & 49.8 & 0.74 \\
Within Europe & 305 & 22.6 & 0.33 \\
Within Africa & 547 & 37.5 & 0.57 \\
Between & 1364 & 65.7 & 0.99 \\
populations & & &
\end{tabular}

Significant differences in expression between strains were determined using a $P$-value cut-off of 0.001 (FDR $=30 \%$ ). 
These comparisons are conservative, because they use a common $P$-value of 0.001 for both sexes, which corresponds to a FDR of $30 \%$ in females, but only $7 \%$ in males. Reducing the FDR in females would reduce the number of polymorphic genes even further. However, even using the minimal $P$-value possible in our analysis $(P=0.0001)$, the FDR does not drop below 20\%. A contributing factor to the observed difference between the sexes may be that there is less statistical power to detect expression polymorphism in the female experiment. Townsend [26] proposed the statistic GEL $_{50}$, which is the fold-change difference at which there is a $50 \%$ chance of detecting a significant difference with $P<$ 0.05 , as a standard for comparing the power of microarray experiments. For the female experiment, the $\mathrm{GEL}_{50}$ was 1.85 . This is higher than the $\mathrm{GEL}_{50}$ of 1.51 reported for the male experiment [18], but still within the range reported for similar surveys of expression polymorphism in Drosophila and other species [2]. However, it is possible that small differences in $\mathrm{GEL}_{50}$ can lead to large differences in the percentage of genes detected as differentially expressed [2].

To investigate the contribution of sex-biased genes to gene expression polymorphism among females, we classified all of the genes on our arrays as male-biased, female-biased, or unbiased using the 5\% FDR meta-analysis of the Sebida database (release 2.0) [27]. Previous studies have shown that male-biased genes are the most polymorphic class of genes when assayed in males $[18,28]$. When assayed in females, there was no significant difference in the level of expression polymorphism among male-biased, female-biased, and unbiased genes (Table 2). However, the general pattern in females followed that in males, with male-biased genes showing

Table 2 Expression polymorphism in sex-biased genes

\begin{tabular}{|c|c|c|c|}
\hline \multirow[b]{2}{*}{ Feature } & \multicolumn{3}{|c|}{ Sex-bias classification } \\
\hline & Female & Male & Unbiased \\
\hline Number of genes on array & 4002 & 2572 & 5988 \\
\hline Percentage of genes detected as expressed & $56.1^{*}$ & $36.5^{*}$ & 44.8 \\
\hline \multicolumn{4}{|l|}{ Percentage of expressed genes: } \\
\hline Polymorphic in Europe & 5.1 & 5.1 & 4.6 \\
\hline Polymorphic in Africa & 8.0 & 9.3 & 8.5 \\
\hline Polymorphic overall & 23.8 & 24.4 & 24.7 \\
\hline $\begin{array}{l}\text { Differentially expressed between } \\
\text { populations }\end{array}$ & $8.4^{+}$ & 10.9 & 11.6 \\
\hline \multicolumn{4}{|l|}{ Average percentage of pairwise differences: } \\
\hline Within Europe & 0.16 & 0.18 & 0.17 \\
\hline Within Africa & 0.25 & 0.32 & 0.30 \\
\hline Overall & 0.65 & 0.87 & 0.79 \\
\hline
\end{tabular}

Genes were classified using the 5\% FDR meta-analysis of the Sebida database [27]. * Significantly different from unbiased genes (FET, $P<0.0001$ ).

${ }^{\dagger}$ Significantly different from male-biased (FET, $\left.P<0.05\right)$ and unbiased (FET, $P<0.001)$ genes. the greatest expression polymorphism and female-biased genes showing the least (Table 2). As expected, there were significant differences in the proportion of genes of different classes that were detected as expressed in females, with $56 \%$ of the female-biased genes and $38 \%$ of the male-biased genes being detected (Table 2). It should be noted that the Sebida sex-bias classifications consider only adult flies raised under standard laboratory conditions and, thus, may overlook genes that show conditiondependent or transient sex-biased gene expression. Baker and Russell [29] identified over 3,500 genes that showed female-biased expression in adult female abdomens during at least one stage of egg development. However, levels of polymorphism in this set of female-biased genes were nearly identical to those in the Sebida female-biased gene set. Of the female-biased genes identified by Baker and Russell [29] that were detected as expressed in our experiment, $23.82 \%(470 / 1,973)$ were polymorphic. The corresponding number for the Sebida female-biased gene set was $23.79 \%(534 / 2,245)$.

It was previously found that, among males, genes residing on the $\mathrm{X}$ chromosome show less expression polymorphism than those residing on the autosomes [18]. This was attributed to the paucity of male-biased genes, which are the most polymorphic class in males, on the X chromosome [18]. Consistent with this interpretation, we found no significant difference in the level of expression polymorphism between X-linked and autosomal genes in females, where many fewer malebiased genes are expressed. The proportions of polymorphic X-linked and autosomal genes were $25.3 \%$ and $23.9 \%$, respectively $\left(\chi^{2}=0.97, P=0.33\right)$. The ratio of $\mathrm{X}$-linked to autosomal significant pairwise differences per gene was 0.96 .

The above results suggest that the difference in expression polymorphism between males and females can be explained partly by sex-biased gene expression, as male-biased genes tend to show the greatest expression polymorphism whether assayed in males or in females [8,28] (Table 2) and make up a much greater proportion of the genes detected as expressed in males. However, when considering only unbiased genes (those expressed nearly equally in males and females), the percentage of polymorphic genes is still 1.6-fold lower in females than in males $\left(24.7 \%\right.$ vs. $39.2 \% ; \chi^{2}=230, P<$ 0.0001). Similarly, unbiased genes show 3.9-fold fewer pairwise differences per gene in females than in males (0.95 vs. 3.74; Mann-Whitney test, $P<0.0001$ ). This suggests that there are general differences between the sexes with respect to the regulation of gene expression and/or the level of purifying selection that restricts gene expression variation.

It has been observed that infection with sigma virus alters the expression of many more genes in males than 
in females [30], which is consistent with male gene expression being more sensitive to genetic and/or environmental perturbations than female gene expression. It has also been shown that genetic variation on the $Y$ chromosome can affect expression levels of many $\mathrm{X}$-linked and autosomal genes $[22,23]$. Thus, one would expect there to be more expression variation among males, as this Y-linked source of expression variation is absent in females. Because our experiments used inbred strains that are homozygous over most of the genome, we are not able to detect gene expression variation caused by non-additive interactions between alleles in heterozygotes. Thus, the level of expression variation measured in our sample may be less than that observed among individuals sampled directly from natural populations. However, since the same inbred lines were used for both the male and female experiments, non-additivity cannot explain the difference observed between the sexes. Previous studies have shown, however, that nonadditive interactions are more prevalent in females than in males [5,21], which suggests that the difference between male and female expression polymorphism might be smaller in natural populations than in comparisons of inbred lines.

\section{Gene expression divergence between populations}

To identify genes that differ in expression between the European and African populations of D. melanogaster, we used data from the 16 microarray hybridizations that directly compared strains of the two populations (indicated by black lines in Figure 1). After quality control, we were able to compare hybridization intensities of 5,584 unique probes, corresponding to 5,370 genes, between the populations. Of these, 569 genes showed a significant inter-population expression difference with $P<0.005$ (FDR = 5\%; Figure 2; Additional file 1). More of the significant genes had higher expression in Europe than in Africa (330 vs. 239; $\left.\chi^{2}=14.6, P<0.0001\right)$. However, the average magnitude of over-expression was greater in Africa than in Europe (1.56-fold vs. 1.37-fold; Mann-Whitney test, $P<0.0001)$. Only two genes showed greater than two-fold over-expression in Europe, while 16 showed greater than two-fold over-expression in Africa (Fisher's exact test (FET), $P<0.0001)$. Similarly, only 34 genes showed greater than 1.5 -fold overexpression in Europe, while 115 showed greater than 1.5 -fold over-expression in Africa (FET, $P<0.0001$ ).

There was not an overrepresentation of sex-biased genes among those showing a significant expression difference between the African and European populations. In fact, there was a slight (but significant) under-representation of female-biased genes among the genes showing differential expression between the populations in females (Table 2). There was also no significant difference in the proportions

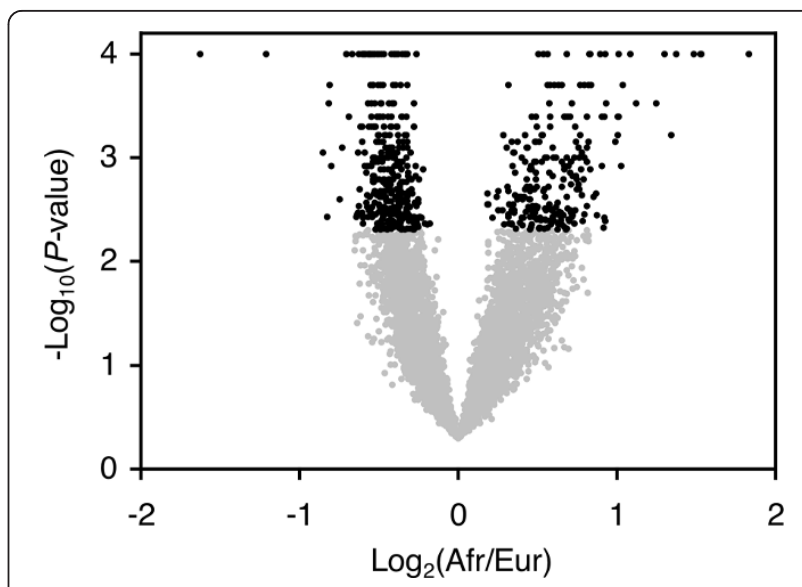

Figure 2 Volcano plot of the between-population analysis. Black points indicate genes with a significant expression difference between the African and European populations of D. melanogaster $(F D R<5 \%)$

of X-linked (10.0\%) and autosomal (10.3\%) genes that showed differential expression between the populations $\left(\chi^{2}=0.10, P=0.76\right)$.

The gene showing the strongest over-expression in the European population was Cyp6g1, a member of the cytochrome P450 gene family that is associated with insecticide resistance [31] (Figure 3). This gene was also found to have the greatest over-expression in male D. melanogaster [18]. Previous studies indicated that high levels of Cyp6g1 expression, which provide increased resistance to DDT and other insecticides, are associated with the insertion of an Accord transposable element upstream of Cyp6g1, as well as with tandem duplication of the Cyp6g1 gene [31-33]. The insertion and duplication are present at high frequency in many non-African populations of D. melanogaster, which has been suggested to be the result of selection for insecticide resistance $[32,33]$. To test for these features in our population samples, we performed PCR on all strains using the previously described diagnostic primers $[31,33]$. The Accord insertion was present in all European strains and in three of the eight African strains. All strains with the Accord insertion, but none of the others, had a tandem duplication of the Cyp6g1 locus (Figure 4; Additional file 2). The three African strains with the insertion/duplication had 2.78-fold higher Cyp6g1 expression than those without (Mann-Whitney test, $P<0.05)$. However, the expression level of the African strains with the insertion/duplication was still 1.57 -fold lower than that of the European strains (Mann-Whitney test, $P<0.05$ ). This suggests that other factors, either cis- or trans-acting, also contribute to the increased Cyp6g1 expression observed for European strains. 

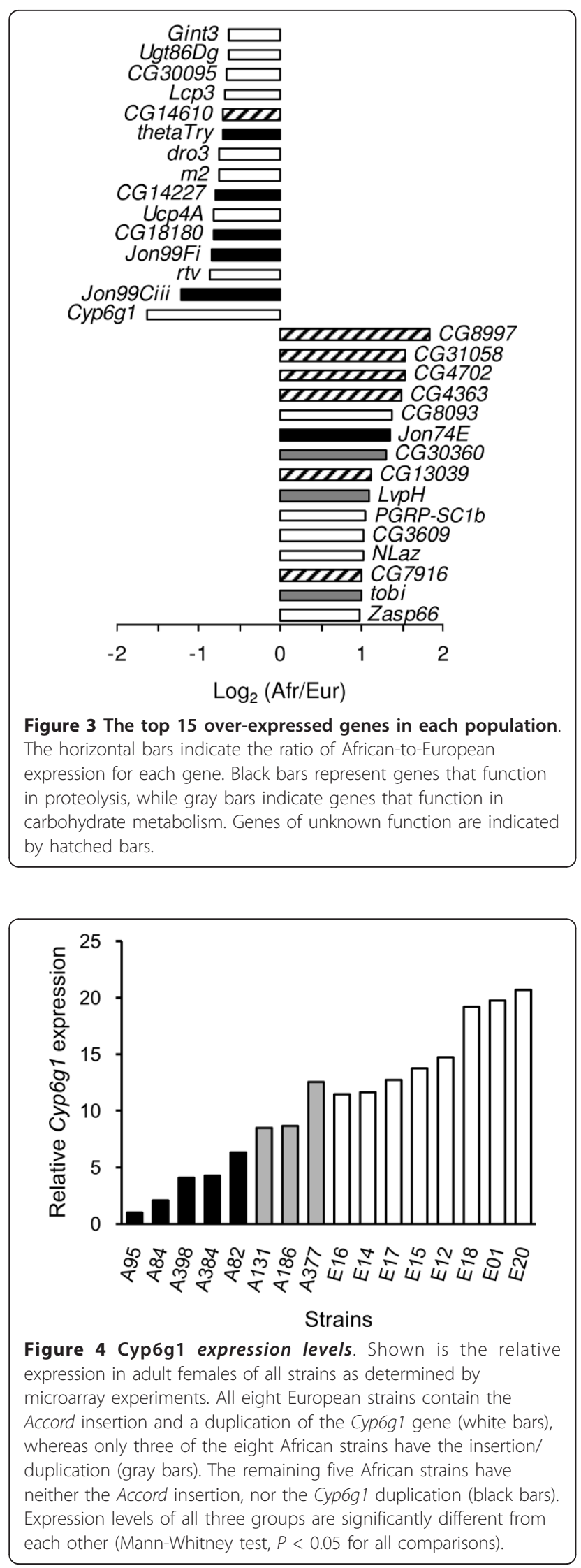

Functional annotation of differentially expressed genes Genes with proteolytic function, particularly serine-type endopeptidases, were consistently over-expressed in the European population (Table 3; Additional file 3). Among the 15 genes with the greatest over-expression in Europe, five function in proteolysis (Figure 3). Of these, the genes with the largest fold-change are members of the Jonah gene family, Jon99Ciii and Jon99Fi, which are serine-type peptidases expressed in the midgut of the adult fly. Other serine-type endopeptidases that were over-expressed in Europe include CG18180, CG14227, and thetaTrypsin (Figure 3). In contrast to the other proteases, one member of the Jonah gene family, Jon 74E, showed significantly higher expression in Africa than in Europe.

Genes involved in sensory perception were enriched in both the Europe over-expressed and Africa overexpressed gene lists. However, the specific pathways differed between the two populations. In Europe, genes involved in olfaction and the detection of chemical stimulus were over-represented (Table 3), while in Africa genes involved in vision and the detection of light stimulus were over-represented (Table 4).

Genes involved in carbohydrate metabolism were also enriched among the genes over-expressed in the African population (Table 4) and several of these genes were among the most over-expressed, including the maltase CG30360, and two $\alpha$-glucosidases $L v p H$ and tobi (Figure 3). tobi has been shown to be a target of the insulin- and glucagon-like signaling system [34]. In this respect, it is noteworthy that the highly over-expressed gene Nlaz, which plays a role in stress response and

Table 3 GO-term enrichment of genes over-expressed in the European population

\begin{tabular}{lllr}
\hline ID & Ontology & Term & $\begin{array}{r}\boldsymbol{P} \text { - } \\
\text { value }\end{array}$ \\
\hline GO:0004984 & MF & Olfactory receptor activity & 0.018 \\
GO:0004252 & MF & Serine-type endopeptidase activity & 0.036 \\
GO:0005337 & MF & $\begin{array}{l}\text { Nucleoside transmembrane } \\
\text { transporter activity }\end{array}$ & 0.039 \\
GO:0035214 & BP & Eye-antennal disc development & 0.001 \\
GO:0008052 & BP & Sensory organ boundary specification & 0.020 \\
GO:0009593 & BP & Detection of chemical stimulus & 0.024 \\
GO:0065004 & BP & Protein-DNA complex assembly & 0.027 \\
GO:0007608 & BP & Sensory perception of smell & 0.027 \\
GO:0009047 & BP & Dosage compensation & 0.033 \\
GO:0001508 & BP & Regulation of action potential & 0.033 \\
GO:0006544 & BP & Glycine metabolic process & 0.033 \\
GO:0008380 & BP & RNA splicing & 0.036 \\
\hline
\end{tabular}

In cases where multiple, related terms within a GO hierarchy were significant, only a single term is given. The complete list is provided in Additional file 3. Ontology abbreviations are: MF, molecular function; BP, biological process. $P$-values were determined by a hypergeometric test with Benjamini-Hochberg multiple-test correction. 
Table 4 GO-term enrichment of genes over-expressed in the African population

\begin{tabular}{|c|c|c|c|}
\hline ID & Ontology & Term & $P$-value \\
\hline GO:0004558 & MF & Alpha-glucosidase activity & 0.006 \\
\hline GO:0004806 & MF & Triglyceride lipase activity & 0.021 \\
\hline GO:0003697 & MF & Single-stranded DNA binding & 0.021 \\
\hline GO:0019201 & MF & Nucleotide kinase activity & 0.021 \\
\hline GO:0004129 & MF & Cytochrome-c oxidase activity & 0.036 \\
\hline GO:0019318 & $\mathrm{BP}$ & Hexose metabolic process & 0.005 \\
\hline GO:0009586 & BP & $\begin{array}{l}\text { Rhodopsin mediated } \\
\text { phototransduction }\end{array}$ & 0.006 \\
\hline GO:0048814 & $\mathrm{BP}$ & $\begin{array}{l}\text { Regulation of dendrite } \\
\text { morphogenesis }\end{array}$ & 0.009 \\
\hline GO:0014866 & BP & Skeletal myofibril assembly & 0.015 \\
\hline GO:0048139 & $\mathrm{BP}$ & Female germ-line cyst encapsulation & 0.015 \\
\hline GO:0035075 & BP & Response to ecdysone & 0.025 \\
\hline GO:0006119 & BP & Oxidative phosphorylation & 0.028 \\
\hline GO:0012502 & BP & Induction of programmed cell death & 0.032 \\
\hline GO:0006631 & BP & Fatty acid metabolic process & 0.035 \\
\hline GO:0007015 & $\mathrm{BP}$ & Actin filament organization & 0.035 \\
\hline GO:0030713 & BP & Ovarian follicle cell stalk formation & 0.036 \\
\hline GO:0016028 & CC & Rhabdomere & 0.018 \\
\hline GO:0044429 & CC & Mitochondrial part & 0.025 \\
\hline GO:0016459 & CC & Myosin complex & 0.025 \\
\hline GO:0030425 & CC & Dendrite & 0.035 \\
\hline
\end{tabular}

In cases where multiple, related terms within a GO hierarchy were significant, only a single term is given. The complete list is provided in Additional file 3. Ontology abbreviations are: MF, molecular function; BP, biological process; CC, cellular compartment. $P$-values were determined by a hypergeometric test with Benjamini-Hochberg multiple-test correction.

determination of adult lifespan, also functions in carbohydrate homeostasis and has been suggested to interfere with insulin signaling [35].

Other enriched functions among the Africa overexpressed genes included oxidative phosphorylation and muscle formation (Table 4). However, many of the Africa over-expressed genes are of unknown function, including six of the 15 genes with the greatest overexpression in Africa and the gene showing the highest overall difference in expression between the African and European populations, CG8997 (Figure 3).

\section{Validation of microarray results by qRT-PCR}

In order to verify the between-population expression differences detected in our microarray analysis, we performed qRT-PCR on a subset of 12 genes, including five with over-expression in Africa, five with overexpression in Europe, and two control genes that showed no difference in expression between populations (Figure 5). For 10 of these genes, including all of those with Africa over-expression, the two control genes, and the three genes with the greatest Europe over-expression, the results were consistent with both

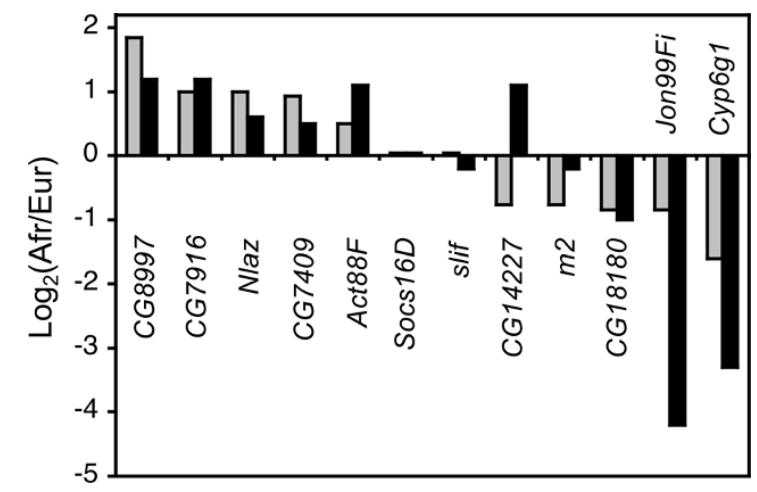

Figure $\mathbf{5}$ Comparison of microarray and qRT-PCR results. Shown is the relative expression difference between the eight African and eight European strains as measured by microarray (gray bars) or qRT-PCR (black bars) for 12 genes. Gene symbols are given below/ above their corresponding values.

methods. One of the genes $(m 2)$ showed strong (1.7fold) Europe over-expression in the microarray experiment, but only slight (1.2-fold) Europe over-expression by qRT-PCR. Another gene (CG14227) showed overexpression in opposite populations when measured by the two methods (Figure 5). The reason for this discrepancy is unclear. It may be because the microarray and qRT-PCR probes match different regions of the CG14227 transcript. However, there is only one annotated transcript for this gene in the current release of FlyBase (release 5.27). When considering all genes and strains, there was a good correlation between expression levels measured by microarray and by qRT-PCR (Pearson's $R=0.5, P<0.0001$; Additional file 4).

\section{Comparison of inter-population gene expression divergence in males and females}

There were many more genes that differed significantly in expression between the European and African populations in females than in males. In females, 10.6\% (569/ 5370 ) of the genes analyzed showed a significant interpopulation difference with a FDR of $5 \%$. In males, $3.4 \%$ $(153 / 4528)$ of the genes analyzed showed a significant inter-population difference with a FDR of $8.7 \%\left(\chi^{2}=\right.$ $189, P<0.0001)$. The lower FDR of the female experiment indicates that this is a conservative comparison. Furthermore, the $\mathrm{GEL}_{50}$ values for the female and male experiments were 1.22 and 1.18 , respectively, indicating that the female experiment had slightly less statistical power to detect differences. This suggests that the different amounts of inter-population gene expression divergence observed between females and males have a biological basis. At the protein level, it has been reported that autosomal female-biased genes show evidence for greater adaptive evolution in the European 
population than in the African population [25]. If this is indicative of a general pattern of stronger selection on females to adapt to the European environment, it could explain the excess of between-population expression differences in females relative to males. A possible reason for this is that females may be under greater selection to survive through the winter, while males that do not survive the winter may still contribute genes to future generations if their sperm is stored in a surviving female [36]. The above hypothesis predicts that most expression differences between populations should be the result of changes occurring within the European population during colonization. At present, we do not have data that would allow us to infer the direction of interpopulation expression changes and test this prediction.

Of the 569 genes identified as differentially expressed between the African and European populations in females and the 153 genes identified as differentially expressed between the same populations in males [18], only 14 genes overlapped (i.e., were significant in both sexes; Table 5). Of these, 12 genes showed higher expression in the same population in both sexes, which is no more than would be expected by chance given the numbers of significant genes in each sex and the total number of genes analyzed in both sexes $\left(\chi^{2}=0.60\right.$, $P=0.44)$. However, several of the overlapping genes (Table 5) represent good candidates for genes that have undergone adaptive regulatory evolution in response to changes in the environment. The gene showing the greatest over-expression in Europe in both males and females was the cytochrome P450 gene Cyp6g1 (see

Table 5 Genes with a significant inter-population expression difference in both females and males

\begin{tabular}{lrrl}
\hline & Log $_{2}$ (Afr/Eur) & \\
\cline { 2 - 3 } Gene & Female & Male* & Function \\
\hline CG8997 & 1.85 & 0.77 & Unknown \\
CG7916 & 1.00 & 0.68 & Unknown \\
CG34330 & 1.00 & 0.38 & Unknown \\
CG7409 & 0.93 & 0.49 & Unfolded protein binding; response to heat \\
retinin & 0.85 & 0.58 & Unknown; expressed in eye \\
CG7953 & 0.85 & 0.49 & Unknown \\
Adk2 & 0.58 & 0.38 & Adenylate kinase; ADP biosynthesis \\
Act88F & 0.49 & 1.54 & Actin filament; indirect flight muscle; \\
& & & immune response \\
Cyp691 & -1.58 & -2.14 & Cytochrome P450; insecticide resistance \\
fau & -0.49 & -0.38 & Unknown; upregulated under anoxia \\
CG17292 & -0.49 & -0.19 & Triglyceride lipase; lipid metabolism \\
CG12262 & -0.38 & -0.38 & Acyl-CoA dehydrogenase; fatty acid beta- \\
& & & oxidation \\
for & 0.51 & -0.31 & cGMP-dependent protein kinase; feeding \\
CG11395 & 0.49 & -0.23 & Unknown \\
\hline
\end{tabular}

*Data from Hutter et al. [18]. above section, Gene expression divergence between populations). The gene CG12262, with an annotated function in oxidation/reduction and fatty acid metabolism, and the gene CG17292, which is also involved in fatty acid metabolism, both showed over-expression in Europe in both sexes. The gene CG7409, which has an annotated function in response to heat and unfolded protein binding, and the actin gene Act88F, which is a component of indirect flight muscle and also involved in the innate immune response, showed consistent African overexpression in both sexes. In addition, three genes of unknown function that are located in a cluster on chromosome arm 2L (CG8997, CG7916, and CG7953) showed significant African over-expression in both males and females (Figure 6). A fourth gene in this cluster, CG33307, showed significant African overexpression in males, but was not detected as expressed in females (Figure 6).

The vast majority of genes detected as being differentially expressed between populations showed this pattern in only one sex. Of the 569 genes that differed in expression between females of the European and African populations, 557 showed this difference only in females. Of these, 310 genes were not detected as expressed in males, while 245 were detected as expressed but their expression did not differ significantly between the populations. Two other genes showed a significant expression difference between populations, but in opposite directions in the two sexes. The first, CG11395, is a gene of unknown function that had significant Africa overexpression in females, but significant Europe overexpression in males. The second is the foraging (for) gene, which encodes a cGMP-dependent protein kinase that influences larval and adult feeding behavior [37-39]. In females, for is significantly over-expressed in Africa, while in males it is significantly over-expressed in Europe.

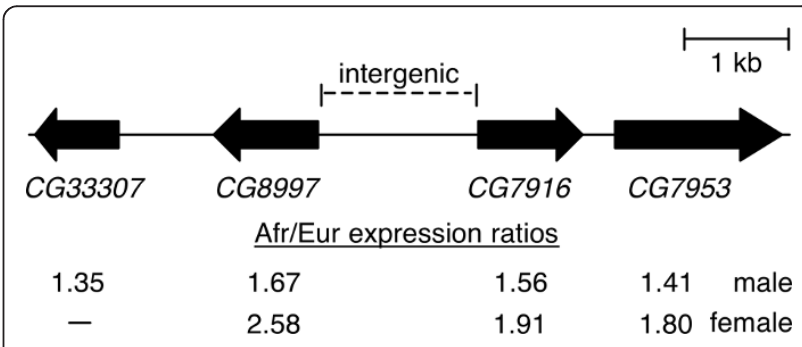

Figure 6 A cluster of genes on chromosome arm 2L with significant over-expression in the African population. Solid boxes represent transcriptional units, with the arrow indicating the direction of transcription. The African/European expression ratio of each gene, as determined by microarray experiments using males [18] and females (present study), is shown at the bottom. Expression of CG33307 was not detected in females. 
To further investigate the effect of sex on interpopulation differences in gene expression, we performed a meta-analysis of the female and male expression data. For each of the 2,315 genes common to both experiments, we determined the difference in mean expression level between the African and European populations, as well as the standard deviation (SD) of this difference, in both females and males. We then calculated the statistic, $d$, by subtracting the Africa-Europe difference in males from that in females and dividing by the pooled SD of the difference in both sexes [40] (Additional file 5). We identified 94 genes for which the difference between males and females was greater than two SD units (FDR $=3.2 \%$ ) and 209 genes for which the difference between males and females was greater than 1.7 SD units (FDR $=5 \%$ ). Of these 209 genes, 176 (84\%) showed enriched expression in opposite populations in the two sexes (Figure 7). There were a few cases in which a gene showed over-expression in the same population in both sexes, but the extent of over-expression was greater in one sex than the other (Figure 7).

\section{Population genetics of a cluster of genes with African over-expression}

The common expression pattern and genomic organization of CG8997, CG7916, and CG7953 suggested that they might share a common regulatory element in the intergenic region between CG8997 and CG7916. To investigate this further, we analyzed DNA sequence

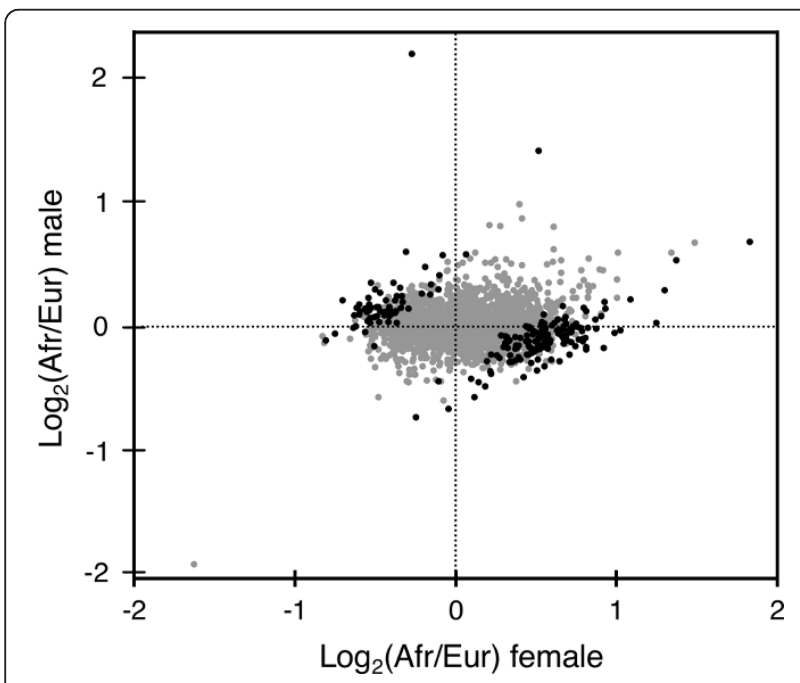

Figure 7 Comparison of inter-population differences in gene expression between males and females. Points represent African/ European expression ratios of the 2315 genes that overlap between the present study and that of Hutter et al. [18]. Black points indicate the 209 genes showing a significant sex-by-population effect (FDR < $5 \%)$. polymorphism in the coding and shared intergenic region of these two genes (Figure 6), which had previously been sequenced in the same strains used in our microarray experiments [41]. In both populations, levels of nucleotide polymorphism $(\pi)$ were normal for a region of moderate recombination. In Africa, values of $\pi$ at synonymous sites in CG8997 and CG7916 and all sites in the intergenic region were $0.028,0.037$, and 0.008, respectively. In Europe, the corresponding values were $0.017,0.040$, and 0.008 . Furthermore, there was no haplotype structure or fixed sequence difference between the populations. Thus, there was no evidence for a recent selective sweep in this region of the genome. The most extreme difference in allele frequency was a single-nucleotide $\mathrm{G} / \mathrm{A}$ polymorphism within the intergenic region in which the derived variant $(G)$ was present in seven of the eight European strains, but only three of the eight African strains. We did, however, find evidence for non-neutral evolution in the coding regions of CG8997 and CG7916, as well as the intergenic region, by the test of McDonald and Kreitman [42] (Table 6). Thus, this region of the genome appears to have been a target of both structural and regulatory adaptation in the past (i.e., since the divergence of D. melanogaster and D. sechellia).

\section{Expression and behavioral divergence between populations}

Previous behavioral studies have shown that there is uni-directional mate-choice preference between $D$. melanogaster strains from Zimbabwe (Z) and cosmopolitan (M) strains, with $\mathrm{Z}$ females showing a preference for $\mathrm{Z}$ males [43,44]. Michalak et al. [45] investigated gene expression in female heads from flies of the two mating types and identified 45 candidate genes that might be involved in the behavioral difference. Only one of these genes (CG7530) was significant in our experiment using whole females. This gene had higher $M$ expression in the experiment of Michalak et al. [45], but higher Zimbabwe expression in our experiment. Thus, there is no concordance between the putative mating-behavior genes expressed differently in $\mathrm{Z}$ and $\mathrm{M}$ female heads

Table 6 Results of McDonald-Kreitman (MK) tests

\begin{tabular}{lcccccccc}
\hline Gene & $\mathbf{D}_{\mathbf{s}}$ & $\mathbf{P}_{\mathbf{s}}$ & $\mathbf{D}_{\mathbf{n}}$ & $\mathbf{P}_{\mathbf{n}}$ & $\mathbf{D}_{\mathbf{i}}$ & $\mathbf{P}_{\mathbf{i}}$ & Nonsynonymous & Intergenic \\
\hline CG8997 & 13 & 16 & 10 & 2 & 71 & 34 & 0.038 & 0.031 \\
CG7916 & 13 & 24 & 6 & 1 & 71 & 34 & 0.032 & 0.008 \\
Combined & 26 & 40 & 16 & 3 & 71 & 34 & $<0.001$ & $<0.001$
\end{tabular}

Shown are the numbers of fixed differences (D) between $D$. melanogaster and $D$. sechellia and the number of polymorphic sites $(P)$ within the African population of $D$. melanogaster. Subscripts indicate synonymous (s), nonsynonymous (n), and intergenic (i) sites. The intergenic region is shared between the two genes (see Figure 6). 
and the genes expressed differently between the Zimbabwe and European populations in whole females. Two of the top candidate genes from Michalak et al. [45], desaturase 2 and Odorant receptor $63 a$ were not detected as being consistently expressed in our experiment and, thus, were excluded from the analysis. However, desaturase 2 expression was detected in a higher proportion of Zimbabwe strains (7/8) than European strains (4/8) and, on average, showed two-fold higher expression in Zimbabwe strains in the hybridizations where it could be detected. This is consistent with the finding of Michalak et al. [45] that desaturase2 shows over-expression in $\mathrm{Z}$ strains. A comparison of gene expression in male heads between a single $Z$ strain and a single $M$ strain uncovered 1216 genes that differed in expression between the mating types [46]. Although only 77 of these genes were detected as differentially expressed between the populations in our analysis, several of the overlapping genes were among those showing the greatest expression difference between Europe and Africa in both sexes, including Cyp6g1, Act88F, and the clustered genes CG8997, CG7916, and CG7953.

\section{Conclusions}

Our microarray survey identified over 500 genes showing low within-population expression polymorphism, but high between-population expression divergence in female D. melanogaster from Europe and Africa. The combination of low polymorphism and high divergence is a hallmark of positive selection and suggests that adaptive evolution at the gene regulatory level has occurred in conjunction with the recent colonization of non-African habitats. This is supported by the finding that Cyp6g1, whose expression is known to play an ecologically relevant role in insecticide resistance, was among the genes with the greatest inter-population expression difference. The functional basis for the interpopulation divergence of the other genes is unknown, however there was an over-representation of genes involved in proteolysis, carbohydrate metabolism and sensory perception (both vision and olfaction). There was very little overlap between genes showing a significant expression difference between populations in females and in males. This suggests that most adaptive changes in gene expression are sex-specific and highlights the need for both sexes to be considered in studies of gene regulatory evolution.

Because our study focused on only one population from the ancestral species range (Zimbabwe) and one from the derived range (the Netherlands), it is not possible to distinguish global "out-of-Africa" adaptations from those that are specific to a local population. Surveys of nuclear DNA polymorphism indicate that there is little population structure within Europe, but more differentiation among some American, Asian, and African populations $[47,48]$. There is also evidence for adaptive evolution of pigmentation, a trait known to be influenced by gene-regulatory variation, among African populations [49]. Thus, there is likely to be gene expression divergence among various African and non-African populations. Further expression studies are needed to investigate this possibility.

\section{Methods \\ Fly strains}

Expression variation was surveyed for eight isofemale strains of both a European (Leiden, the Netherlands) and an African (Lake Kariba, Zimbabwe) population of D. melanogaster. The populations are as described in Glinka et al. [16]. The fly strains were the same as those used in the expression analysis of adult male flies by Hutter et al. [18]. Flies were maintained on standard cornmeal-molasses medium at $22^{\circ}$ and constant lighting.

\section{Microarray platform}

The CDMC 14kv1 microarray (Canadian Drosophila Microarray Centre, Mississauga, Canada) was used for all hybridizations. This platform features a total of 32,448 oligonucleotide probes (65-69 bases), each spotted in duplicate. The probes represent 13,688 unique genes, which correspond to $92 \%$ of those in the current D. melanogaster genome annotation (FlyBase release 5.27). Since the transcript-specific probes were designed to release 4.1 of the genome, some genes in the current annotation are not represented on the array, whereas others are represented by more than one probe.

\section{RNA extraction, hybridization, and scanning}

For each strain, total RNA of 40 mated female flies, four-to-six days of age, was extracted using TRIzol reagent (Invitrogen, Carlsbad, CA, USA) and samples were stored at $-80^{\circ}$. Reverse transcription was conducted using $50 \mu \mathrm{g}$ of total RNA per strain and anchored oligo (dT) primers. cDNA samples were labeled with Alexa Flour 555 and 647 dyes using the SuperScript Plus Indirect cDNA Labeling System (Invitrogen) and following the manufacturer's protocol.

To compare expression levels of all fly strains to each other, the hybridization scheme developed by Hutter et al. [18] was followed. This approach allows expression levels of all strains to be compared, while keeping the number of hybridizations at a practical level (Figure 1). Six or eight replicate hybridizations per strain were performed on a total 56 microarrays. For each strain, three or four competitive hybridizations with other strains, plus their respective dye-swap hybridizations were performed. For technical replicates (dye-swaps), RNA from the same extractions was used, whereas for biological 
replicates (different pairwise hybridizations of strains), RNA extracted from a new set of flies was used. Arrays were pre-hybridized and washed using the Pronto! Universal Microarray Kit (Corning, Lowell, MA, USA) according to the manufacturer's protocol. Otherwise, hybridizations were conducted following the CDMC protocol. Arrays were scanned with an aQuire 2-laser microarray scanner and Qscan software (Genetix, New Milton, UK). All microarray data have been submitted to the Gene Expression Omnibus database under the accession numbers GSM580470-GSM580525 (platform GPL3603, series GSE23662).

\section{Microarray data analysis}

Raw fluorescence intensities were normalized using CARMAweb [50], which is a web-based interface to the 'limma' package of Bioconductor [51]. The default settings of 'minimum', 'printtiploess', and 'quantile' were used for background correction, within-array normalization, and between-array normalization, respectively. Between-array normalization was done using pairs of dye-swap hybridizations. As a quality control step to eliminate background noise from genes that are not expressed (or expressed only at very low levels) in adult females, we required that a spot have mean signal intensity at least one SD above local background in both channels to be included in the analysis. In cases where both replicate spots of a probe passed quality control, the arithmetic mean of their $\log _{2}$ (red/green) intensities was used. Otherwise, only the red/green intensity of the spot passing quality control was used.

The resulting normalized red/green-ratios were used as input for BAGEL [52], a program that estimates relative expression levels for each gene in each of the 16 strains using a Bayesian framework. To determine the experiment-wide false discovery rate (FDR), we repeated the BAGEL analysis on a randomized version of our final data set. Randomization was performed by sampling- with-replacement within each hybridization (i.e., randomizing within a column), thereby maintaining the underlying data structure (e.g., excluded genes) within each hybridization. The resulting output was used to determine the FDR corresponding to a given $P$-value.

To identify genes that differ in expression between Africa and Europe on a population level, we repeated the BAGEL analysis using only the 16 hybridizations in which an African strain was compared directly to a European strain (black lines in Figure 1). All strains from the same population were combined into a single node and, thus, treated as biological replicates from within a population. To determine the FDR, BAGEL was run on a randomized data set that was created by permuting the expression ratios of the replicate hybridizations within each gene (i.e., randomizing within a row). As an additional quality control step, we required that each gene be detected as expressed (by the criteria described above) in at least nine of the 16 replicate hybridizations.

\section{qRT-PCR}

For each strain, qRT-PCR of two biological replicates, representing two separate RNA extractions of 20 four-tosix day-old mated female flies, was performed. Following DNase I digestion, $5 \mu \mathrm{g}$ of total RNA was reverse transcribed using Superscript II reverse transcriptase and random hexamer primers (Invitrogen). The resulting cDNA was diluted 1:40 and used for qRT-PCR with TaqMan probes and TaqMan Gene Expression Master Mix (Applied Biosystems, Foster City, CA, USA) according to the manufacturer's protocol. To validate expression differences detected by our microarray analysis, qRT-PCR was performed on a Bio-Rad Real-Time thermal cycler CFX96 (Bio-Rad, Hercules, CA, USA) for the following target genes (TaqMan IDs are given in parentheses): Cyp $6 g 1$ (Dm01819889_g1), Jon99Fi (Dm02146518_s1), CG18180 (Dm01801887_s1), CG14227 (Dm01845429_g1), m2 (Dm02151465_s1), CG8997 (Dm01791303_g1), CG7916 (Dm01791305_g1), Nlaz (Dm01844577_g1), CG7409 (Dm01840751_s1), Act88F (Dm02362815_s1), CG18179 (Dm01801878_s1), slif (Dm01792789_g1), and Socs16D (Dm01813854_g1). Expression levels of all target genes were normalized to Actin 5C (Dm02361909_s1), which was used as an internal control. All assays were performed in three technical replicates, and for each gene the average threshold cycle $(\mathrm{Ct})$ value over all biological and technical replicates was determined. $\Delta \mathrm{Ct}$ values were calculated by subtracting the control $\mathrm{Ct}$ from the target $\mathrm{Ct}$ value. The fold-change in expression between two samples was calculated as $2^{(-\Delta \mathrm{Ct} 1-\Delta \mathrm{Ct} 2)}$. To determine the fold-change between the African and the European population, $\Delta \mathrm{Ct}$ values were averaged among strains within each population and the European value was used as $\Delta \mathrm{Ct}_{2}$.

\section{GO analysis}

Enriched GO terms within the lists of differentially expressed genes were identified using the GOEAST web server [53]. Prior to analysis, the annotation of the CDMC microarrays was updated to match FlyBase release 5.27 by performing a BLAT search of all probe sequences with the UCSC genome browser [54]. Probes giving a unique hit to an annotated transcript were matched with their release 5.27 GO terms. Significant GO term enrichment was determined by the hypergeometric method with Hochberg FDR multiple-test correction [55], with the FDR set to 0.05. As a background for GO enrichment tests, we used all genes on the CDMC microarray that were detected as expressed in our experiments (i.e., those passing the quality control steps described above). 


\section{DNA sequence analysis}

DNA sequence polymorphism in the genomic region encompassing the genes CG8997 and CG7916 was previously reported [41]. These authors directly sequenced PCR-amplified genomic DNA from the same strains used in our microarray analysis, plus an additional four strains each from the Zimbabwe and Netherlands populations [16]. We used all of the available sequences for McDonald-Kreitman tests [42] of selection on nonsynonymous and intergenic sites using the DnaSP (v5) software [56]. The test compares ratios of divergenceto-polymorphism at the test sites (nonsynonymous or intergenic) to those at synonymous sites and provides evidence for adaptive evolution when there is a relative excess of divergence at the test sites, which is consistent with recurrent selective sweeps since the time of speciation.

To analyze sequence variation in the Cyp6g1 region, we performed diagnostic PCR on the 16 strains used in our microarray analysis. The primers used to detect the Accord insertion were 5'-GAAAGCCGGTTGTGTTTAATTAT-3' and 5'-CTTTTTGTGTGCTATGGTTTAGTTAG-3', which flank the insertion site. An additional forward primer complementary to the Accord insertion (5'-GGGTGCAACAGAGTTTCAGGTA-3') was used to confirm its presence [31]. The primers used to detect tandem duplication of the Cyp $6 g 1$ locus were 5'-CGAGTACGAGAGCGTGGAG-3' and 5'-ATTAAACACAACCGGCTTTCTCG-3' [33]. Following PCR, the products were sequenced to confirm that the expected target sequence was amplified.

\section{Statistical analysis}

For comparisons of categorical data (e.g., numbers of polymorphic and non-polymorphic genes in males and females) we used standard $2 \times 2$ contingency table analyses. $P$-values were determined by Fisher's exact test or, when the sample sizes were large, by a chi-squared approximation. To test for differences between two samples (e.g., Cyp6g1 expression between strains with and without the Accord insertion) we used the non-parametric Mann-Whitney $U$ test, which compares the rank-sums of the observed values of two samples. This approach was used to avoid making assumptions about the underlying distribution of gene expression levels among individuals or classes of genes. All tests were performed using R (version 2.10.1) [57].

\section{Additional material}

Additional file 1: Expression divergence between the African and European populations. Table of relative expression levels in the African and European populations of all genes used in the analysis.
Additional file 2: Diagnostic PCR for the Accord insertion and tandem duplication of the Cyp6g1 gene. Agarose gel images of diagnostic PCR for the Accord element insertion and tandem duplication of the Cyp6g1 gene.

Additional file 3: GO-term enrichment of genes over-expressed in the African and European populations. Table of all GO-terms with significant over-representation in each population.

Additional file 4: Correlation of fold-change expression differences as measured by microarray and qRT-PCR. Plot of 1,560 pairwise comparisons of all $16 \mathrm{D}$. melanogaster strains for 13 different genes. Additional file 5: Meta-analysis of male and female betweenpopulation gene expression divergence. Table of differences in expression between the African and European populations for all genes common to the female and male experiments.

\section{Acknowledgements}

We thank Hedwig Gebhart and Carmen lannitti for technical assistance and all members of the Munich population genetics group for comments and discussion. This work was carried out as part of the research unit "Natural selection in structured populations" (FOR 1078) funded by Deutsche Forschungsgemeinschaft grants STE 325/12-1 to WS and PA 903/5-1 to JP. RS was supported by a fellowship from the international research training program "RECESS: Regulation and evolution of cellular systems" (Deutsche Forschungsgemeinschaft GRK 1563).

\section{Author details}

'Department of Biology II, University of Munich (LMU), 82152 PlaneggMartinsried, Germany. ${ }^{2}$ Department of Systems Biology, Harvard Medical School, Boston, Massachusetts, USA.

\section{Authors' contributions}

LM performed the microarray and qRT-PCR experiments. SH, RS and JP analyzed the microarray data. LM and SSS performed the population genetic analysis of DNA sequences. WS and JP conceived of the study, and participated in its design and coordination. LM and JP drafted the manuscript with input from all authors. All authors read and approved the final manuscript.

Received: 5 November 2010 Accepted: 28 January 2011 Published: 28 January 2011

\section{References}

1. Whitehead A, Crawford DL: Variation within and among species in gene expression: raw material for evolution. Mol Ecol 2006, 15:1197-1211.

2. Clark TA, Townsend JP: Quantifying variation in gene expression. Mol Ecol 2007, 16:2613-2616.

3. Ellegren $\mathrm{H}$, Parsch J: The evolution of sex-biased genes and sex-biased gene expression. Nat Rev Genet 2007, 8:689-698.

4. Jin W, Riley RM, Wolfinger RD, White KP, Passador-Gurgel G, Gibson G: The contributions of sex, genotype and age to transcriptional variance in Drosophila melanogaster. Nat Genet 2001, 29:389-395.

5. Gibson G, Riley-Berger R, Harshman L, Kopp A, Vacha S, Nuzhdin S, Wayne M: Extensive sex-specific nonadditivity of gene expression in Drosophila melanogaster. Genetics 2004, 167:1791-1799.

6. Ayroles JF, Carbone MA, Stone EA, Jordan KW, Lyman RF, Magwire MM, Rollmann SM, Duncan LH, Lawrence F, Anholt RRH, Mackay TF: Systems genetics of complex traits in Drosophila melanogaster. Nat Genet 2009, 41:299-307.

7. Wayne ML, Pan YJ, Nuzhdin SV, Mclntyre LM: Additivity and trans-acting effects on gene expression in male Drosophila simulans. Genetics 2004, 168:1413-1420.

8. Baker DA, Meadows LA, Wang J, Dow JA, Russell S: Variable sexually dimorphic gene expression in laboratory strains of Drosophila melanogaster. BMC Genomics 2007, 8:454.

9. David JR, Capy P: Genetic variation of Drosophila melanogaster natural populations. Trends Genet 1988, 4:106-111. 
10. Haddrill PR, Thornton KR, Charlesworth B, Andolfatto P: Multilocus patterns of nucleotide variability and the demographic and selection history of Drosophila melanogaster populations. Genome Res 2005, 15:790-799.

11. Ometto L, Glinka S, De Lorenzo D, Stephan W: Inferring the effects of demography and selection on Drosophila melanogaster populations from a chromosome-wide scan of DNA variation. Mol Biol Evol 2005, 22:2119-2130

12. Thornton $K$, Andolfatto P: Approximate Bayesian inference reveals evidence for a recent, severe bottleneck in a Netherlands population of Drosophila melanogaster. Genetics 2006, 172:1607-1619.

13. Stephan W, Li H: The recent demographic and adaptive history of Drosophila melanogaster. Heredity 2007, 98:65-68.

14. Townsend JP, Rand DM: Mitochondrial genome size variation in New World and Old World populations of Drosophila melanogaster. Heredity 2004, 93:98-103.

15. Harr B, Kauer M, Schlötterer C: Hitchhiking mapping: a population-based fine-mapping strategy for adaptive mutations in Drosophila melanogaster. Proc Natl Acad Sci USA 2002, 99:12949-12954.

16. Glinka S, Ometto L, Mousset S, Stephan W, De Lorenzo D: Demography and natural selection have shaped genetic variation in Drosophila melanogaster: a multi-locus approach. Genetics 2003, 165:1269-1278.

17. Li H, Stephan W: Inferring the demographic history and rate of adaptive substitution in Drosophila. PLoS Genet 2006, 2:e166.

18. Hutter S, Saminadin-Peter SS, Stephan W, Parsch J: Gene expression variation in African and European populations of Drosophila melanogaster. Genome Biol 2008, 9:R12.

19. Parisi M, Nuttall R, Naiman D, Bouffard G, Malley J, Andrews J, Eastman S, Oliver B: Paucity of genes on the Drosophila X chromosome showing male-biased expression. Science 2003, 299:697-700.

20. Ranz JM, Castillo-Davis Cl, Meiklejohn CD, Hartl DL: Sex-dependent gene expression and evolution of the Drosophila transcriptome. Science 2003, 300:1742-1745.

21. Wayne ML, Telonis-Scott M, Bono LM, Harshman L, Kopp A, Nuzhdin SV, Mclntyre LM: Simpler mode of inheritance of transcriptional variation in male Drosophila melanogaster. Proc Natl Acad Sci USA 2007, 104:18577-18582.

22. Lemos B, Araripe LO, Hartl DL: Polymorphic Y chromosomes harbor cryptic variation with manifold functional consequences. Science 2008, 319:91-93.

23. Jiang PP, Hartl DL, Lemos B: Y not a dead end: epistatic interactions between $Y$-linked regulatory polymorphisms and genetic background affect global gene expression in Drosophila melanogaster. Genetics 2010, 186:109-118.

24. Hutter S, Li H, Beisswanger S, De Lorenzo D, Stephan W: Distinctly different sex ratios in African and European populations of Drosophila melanogaster inferred from chromosomewide single nucleotide polymorphism data. Genetics 2007, 177:469-480.

25. Parsch J, Zhang Z, Baines JF: The influence of demography and weak selection on the McDonald-Kreitman test: an empirical study in Drosophila. Mol Biol Evol 2009, 26:691-698.

26. Townsend JP: Resolution of large and small differences in gene expression using models for the Bayesian analysis of gene expression levels and spotted DNA microarrays. BMC Bioinformatics 2004, 13:1-13.

27. Gnad F, Parsch J: Sebida: a database for the functional and evolutionary analysis of genes with sex-biased expression. Bioinformatics 2006, 22:2577-2579.

28. Meiklejohn CD, Parsch J, Ranz JM, Hartl DL: Rapid evolution of malebiased gene expression in Drosophila. Proc Natl Acad Sci USA 2003, 100:9894-9899.

29. Baker DA, Russell S: Gene expression during Drosophila melanogaster egg development before and after reproductive diapause. BMC Genomics 2009, 10:242.

30. Carpenter J, Hutter S, Baines JF, Roller J, Saminadin-Peter SS, Parsch J, Jiggins FM: The transcriptional response of Drosophila melanogaster to infection with the sigma virus (Rhabdoviridae). PLoS One 2009, 4:e6838.

31. Daborn PJ, Yen JL, Bogwitz MR, Le Goff G, Feil E, Jeffers S, Tijet N, Perry T, Heckel D, Batterham P, Feyereisen R, Wilson TG, ffrench-Constant RH: A single p450 allele associated with insecticide resistance in Drosophila. Science 2002, 297:2253-2256.

32. Catania F, Kauer MO, Daborn PJ, Yen JL, Ffrench-Constant RH, Schlotterer C: World-wide survey of an Accord insertion and its association with DDT resistance in Drosophila melanogaster. Mol Ecol 2004, 13:2491-2504.

33. Schmidt JM, Good RT, Appleton B, Sherrard J, Raymant GC, Bogwitz MR Martin J, Daborn PJ, Goddard ME, Batterham P, Robin C: Copy number variation and transposable elements feature in recent, ongoing adaptation at the Cyp6g1 locus. PLoS Genet 2010, 6:e1000998.

34. Buch S, Melcher C, Bauer M, Katzenberger J, Pankratz MJ: Opposing effects of dietary protein and sugar regulate a transcriptional target of Drosophila insulin-like peptide signaling. Cell Metab 2008, 7:321-332.

35. Hull-Thompson J, Muffat J, Sanchez D, Walker DW, Benzer S, Ganfornina MD, Jasper $\mathrm{H}$ : Control of metabolic homeostasis by stress signaling is mediated by the lipocalin NLaz. PLoS Genet 2009, 5: e1000460.

36. Collett Jl, Jarman MG: Adult female Drosophila pseudoobscura survive and carry fertile sperm through long periods in the cold: populations are unlikely to suffer substantial bottlenecks in overwintering. Evolution Int J Organic Evolution 2001, 55:840-845.

37. de Belle JS, Hilliker AJ, Sokolowski MB: Genetic localization of foraging (for): a major gene for larval behavior in Drosophila melanogaster. Genetics 1989, 123:157-163.

38. Pereira HS, Sokolowski MB: Mutations in the larval foraging gene affect adult locomotory behavior after feeding in Drosophila melanogaster. Proc Natl Acad Sci USA 1993, 90:5044-5046.

39. Shaver SA, Varnam CJ, Hilliker AJ, Sokolowski MB: The foraging gene affects adult but not larval olfactory-related behavior in Drosophila melanogaster. Behav Brain Res 1998, 95:23-29.

40. Cohen J: Statistical power analysis for the behavioral sciences. 2 edition. Lawrence Erlbaum Associates, Hillsdale, NJ; 1988.

41. Parsch J, Novozhilov S, Saminadin-Peter SS, Wong KM, Andolfatto P: On the utility of short intron sequences as a reference for the detection of positive and negative selection in Drosophila. Mol Biol Evol 2010, 27:1226-1234.

42. McDonald $\mathrm{JH}$, Kreitman M: Adaptive protein evolution at the Adh locus in Drosophila. Nature 1991, 351:652-654

43. Wu Cl, Hollocher H, Begun DJ, Aquadro CF, Xu Y, Wu ML: Sexual isolation in Drosophila melanogaster: a possible case of incipient speciation. Proc Natl Acad Sci USA 1995, 92:2519-2523.

44. Hollocher H, Ting CT, Wu ML, Wu Cl: Incipient speciation by sexual isolation in Drosophila melanogaster: extensive genetic divergence without reinforcement. Genetics 1997, 147:1191-1201.

45. Michalak P, Malone JH, Lee IT, Hoshino D, Ma D: Gene expression polymorphism in Drosophila populations. Mol Ecol 2007, 16:1179-1189.

46. Wang HY, Fu Y, McPeek MS, Lu X, Nuzhdin S, Xu A, Lu J, Wu ML, Wu Cl: Complex genetic interactions underlying expression differences between Drosophila races: analysis of chromosome substitutions. Proc Natl Acad Sci USA 2008, 105:6362-6367.

47. Pool JE, Aquadro CF: History and structure of sub-Saharan populations of Drosophila melanogaster. Genetics 2006, 174:915-929.

48. Nunes MD, Neumeier H, Schlötterer C: Contrasting patterns of natural variation in global Drosophila melanogaster populations. Mol Ecol 2008, 17:4470-4479.

49. Pool JE, Aquadro CF: The genetic basis of adaptive pigmentation variation in Drosophila melanogaster. Mol Ecol 2007, 16:2844-2851.

50. Rainer J, Sanchez-Cabo F, Stocker G, Sturn A, Trajanoski Z: CARMAweb: comprehensive R- and bioconductor-based web service for microarray data analysis. Nucleic Acids Res 2006, 34:W498-503.

51. Gentleman RC, Carey VJ, Bates DM, Bolstad B, Dettling M, Dudoit S, Ellis B, Gautier L, Ge Y, Gentry J, Hornik K, Hothorn T, Huber W, lacus S, Irizarry R, Leisch F, Li C, Maechler M, Rossini AJ, Sawitzki G, Smith C, Smyth G, Tierney L, Yang JY, Zhang J: Bioconductor: open software development for computational biology and bioinformatics. Genome Biol 2004, 5:R80.

52. Townsend JP, Hartl DL: Bayesian analysis of gene expression levels: statistical quantification of relative mRNA level across multiple strains or treatments. Genome Biol 2002, 3:RESEARCH0071.

53. Zheng Q, Wang XJ: GOEAST: a web-based software toolkit for Gene Ontology enrichment analysis. Nucleic Acids Res 2008, 36:W358-363.

54. Rhead B, Karolchik D, Kuhn RM, Hinrichs AS, Zweig AS, Fujita PA, Diekhans M, Smith KE, Rosenbloom KR, Raney BJ, Pohl A, Pheasant M, Meyer LR, Learned K, Hsu F, Hillman-Jackson J, Harte RA, Giardine B, Dreszer TR, Clawson H, Barber GP, Haussler D, Kent WJ: The UCSC genome browser database: update 2010. Nucleic Acids Res 2010, 38:D613-619. 
55. Benjamini $Y$, Hochberg $Y$ : Controlling the false discovery rate: a practical and powerful approach to multiple testing. J Royal Stat Soc B 1995, 57:289-300.

56. Librado P, Rozas J: DnaSP v5: a software for comprehensive analysis of DNA polymorphism data. Bioinformatics 2009, 25:1451-1452.

57. R Development Core Team: R: A language and environment for statistical computing, reference index version 2.2.1. Vienna, Austria: R Foundation for Statistical Computing; 2005.

doi:10.1186/1471-2164-12-81

Cite this article as: Müller et al:: Population transcriptomics of Drosophila melanogaster females. BMC Genomics 2011 12:81.

Submit your next manuscript to BioMed Central and take full advantage of:

- Convenient online submission

- Thorough peer review

- No space constraints or color figure charges

- Immediate publication on acceptance

- Inclusion in PubMed, CAS, Scopus and Google Scholar

- Research which is freely available for redistribution

Submit your manuscript at www.biomedcentral.com/submit
C Biomed Central 